\section{Stretching of a vortical}

\section{structure: filaments of}

\section{vorticity'}

Philippe Petitjeans, Laboratoire de Physique et de Mécanique des Milieux Hétérogènes, Ecole Supérieure de Physique et de Chimie Industrielles, Paris, France

Tortical structures are commonly formed in flows: One has noticed a bath tub vortex, or a vortex formed with a kitchen mixer. The largest vortical structures can be observed in Earth's high-atmospheric flows where cyclones or anti-cyclones can reach up to $1000 \mathrm{~km}$ diameter with typical velocities of the order of $100 \mathrm{~km} / \mathrm{h}$. Large structures can also be seen in oceans with typical diameters of $100 \mathrm{~km}$ with velocities of few tens of $\mathrm{km} / \mathrm{h}$. On the other side of the spectrum, the smallest vortices also have a limit, which is not due to the size of the planet, but to viscosity: below a certain size, viscous dissipation does not allow for an organized coherent structure. Its whole energy is dissipated as heat in the flow. On an intermediate scale, tornados are spectacular illustrations of vortical structures or vortices (Fig. 1).

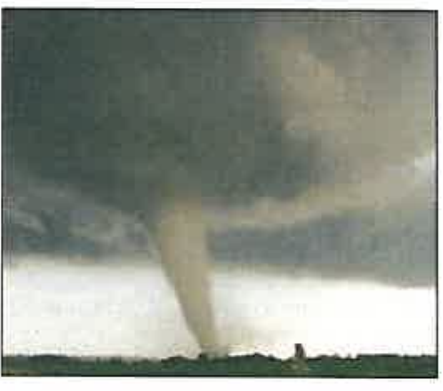

A Fig. 1: Picture of a tornado.
Intituively, one understands that a vortex can be described by two parameters: Its size, and its circumferential velocity. The size of the core of the vortex is defined as the distance $r_{0}$ from the vortex axis where the azimuthal velocity $V_{\theta}$ is maximal $V_{\theta}\left(r_{0}\right)=V_{\theta \max }$. For distances larger than $r_{0}$, the rotationel velocity decreases and goes to zero at "infinity". Below this diameter $2 r_{0}$, the vortex is approximately in a state of solid-body rotation, as shown in Figure 2a.

Another important parameter is the vorticity $\omega$ which is the curl of the velocity $\omega=\operatorname{rot} V$. Alternatively, it can be written as twice the angular velocity $\Omega$ of the vortex. In the case represented in Figure 2a, the vorticity has only a non-zero component in the direction along the vortex axis. This vorticity is represented in Figure $2 \mathrm{~b}$.
In this example, one observes indeed that the vorticity is concentrated in the vortex core. A flow with $V_{\theta} \sim 1 / r$ outside the core has been chosen. This gives a zero vorticity outside this region. Note that such a velocity field depends on $r$ although its axial vorticity is zero: $\omega_{z}=1 / r\left[\partial / \partial r\left(V_{\theta} / r\right) r-r \partial V_{r} / \partial \theta\right]=0$. In a similar way, a non-zero vorticity does not mean that a vortex is present: $A$ shear flow such as a boundary layer flow ${ }^{2}$ on a flat wall can be stable until large Reynolds number $(\operatorname{Re}=U L / v)$. For all vortical flows, vorticity is a very important parameter since it locates vortices and gives their "intensity".

An important mechanism that enhances the vorticity is the stretching. Stretching a vortex along its axis will make it rotate faster and decrease its diameter in order to maintain its kinetic momentum constant. This is analogous to the kinetic momentum conservation law in solid mechanics. A well known example is the ice-skater who turns faster as she brings her hands near her body, and vice versa. An example in fluid mechanics is the bath-tub vortex that rotates faster and becomes smaller as it goes from the fluid surface to the exit. More precisely, a stretching $\gamma$ is an acceleration of the axial velocity along the vortex $(\gamma=\nabla V)$. The vorticity equation is obtained from the curl of the Navier-Stokes equation:

$$
\partial \omega / \partial t+(\mathrm{V} . \nabla) \omega=(\omega . \nabla) \mathrm{V}+v \Delta \omega .
$$

The first term represents the temporal evolution of the vorticity, the second is an advection term, the third is the stretching term and the fourth represents the viscous effects. When stretching is parallel and in the same direction as the vorticity, the term $(\omega . \nabla)$ is positive and amplifies the vorticity $(\partial \omega / \partial t)$. The vorticity increases and the viscous term $(v \Delta \omega)$ becomes large enough to exactly counter balance the amplification term $(\omega . \nabla)$ V. An equilibrium is then reached which imposes the diameter of the vortex $\gamma . \omega=v \omega / r_{0}^{2}$, hence $r_{0} \approx(v / \gamma)^{1 / 2}$. From the general perspective of view, the stretching of the vorticity in the flow leads to its amplification and its confinement. Note that it is not the vorticity which is conserved, since it is amplified by stretching, but the circulation $\Gamma=0 \int_{\mathrm{c}} U$.dl. Circulation around a curve $\mathrm{C}$ is the total vorticity inside this curve.

Vortex stretching is a very important mechanism in fluid dynamics: it usually corresponds to the presence of vortical structures which are much more intense that those produced by simple shear or rotating flows. In particular, it is now well known that local stretching of vorticity in turbulent flows produces very intense vortices called "filaments of vorticity". A large part of the scientific community working on turbulence believes that these structures are very important in the dynamics of turbulent flows, although their structure, their dynamics or their instabilities are not fully understood. The study of these structures of intense vorticity is hence extremely important for both fundamental research
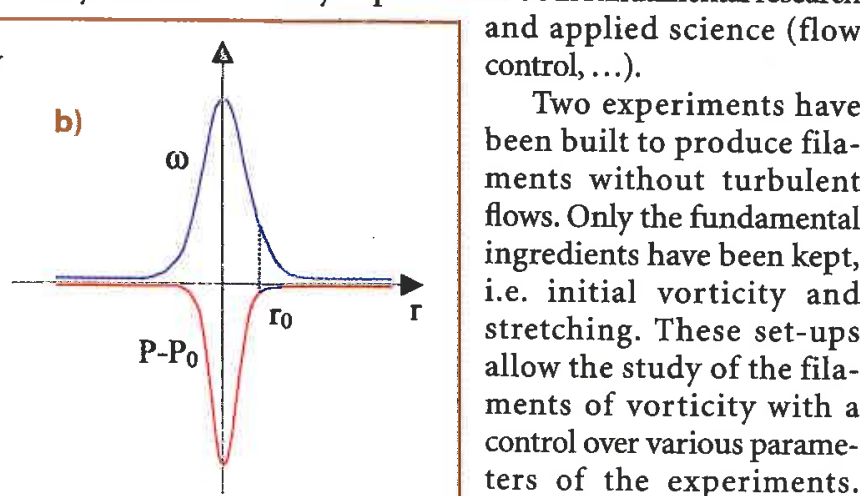
control,....).

Two experiments have been built to produce filaments without turbulent flows. Only the fundamental ingredients have been kept, i.e. initial vorticity and stretching. These set-ups allow the study of the filaments of vorticity with a control over various parameters of the experiments. In that way, an isolate 
"standard" vortex is produced for a study of its structure, its dynamics, its instabilities and its turbulent burst.

The two experiments allow a direct study of the mechanisms behind a vortex structure along with its dynamics. Note that the vortex structure is much more complex than models such as Burgers'vortex.

The first experiment corresponds to the stretching of the vorticity of a laminar boundary layer flow in a water channel. The second produces a stretched vortex between two co-rotating discs. In the latter experiment, filaments of vorticity of stronger intensity are produced as the injected vorticity is much larger with rotating discs than with a boundary layer flow. These two experiments cover a large range of vorticity.

\section{Experiment in a water channel (stretching of a boundary layer)}

A vortex is generated by stretching the vorticity of a stable laminar boundary layer flow which, under natural conditions, does not produce a vortex. The initial vorticity sheet occurs in the laminar boundary layer $\left(\operatorname{Re}_{\delta} \approx 100\right)$. Figure 3 shows a sketch of the channel section where the study is performed. On the right hand side of the figure; the flow develops boundary layers on each wall. The boundary layer on the lower plate has, for instance, a nonzero component of the vorticity $(\partial U / \partial y \neq 0)$. This "initial" vorticity $\omega_{i}$ will be artificially enhanced to a powerful single vortex. To enable this, a stretching, along this initial vorticity is carried out through suction from slots located in lateral walls. The suction generate a stretching, i.e. an acceleration or a transversal velocity gradient $\nabla V$, parallel to the initial vorticity $\omega_{i}$. When the stretching is large enough, a strong vortex is produced between the two suction slots.

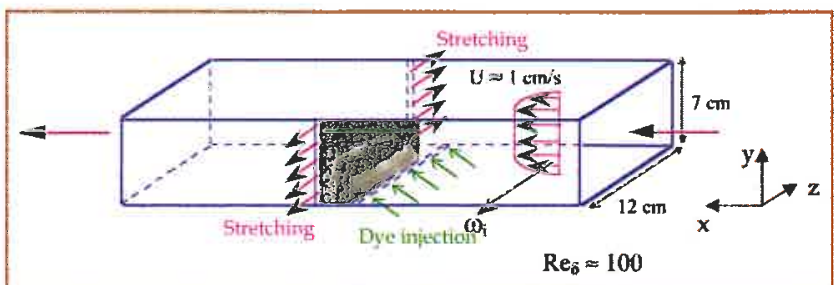

A Fig. 3: Experimental set-up.

As the mean flow is laminar and the velocities are slow (0 to 10 $\mathrm{cm} / \mathrm{s}$ ), the study of a stretched vortex becomes easier compared to a study in a turbulent flow. Some examples of flow visualization are shown in photographs of Figures 4 , which were obtained from either the injection of fluorescent dye in conjunction with argon laser lighting or from the injection of food colour dye upstream.

If the whole flow goes through the suction, a permanent vortex is produced. In that case, its structure is studied with velocity measurements as well as its eventual instabilities. If only a part of the flow goes through the suction slots while the rest goes downstream (on the left hand side of the Figure 3), the vortex tends to be advected by the flow while the suction tends to keep it attached. Depending on the ratio between these two mechanisms, the vortex can remain attached or can explode as a turbulent spot. The vortex cannot persist after detachment from suction slots since it lost its stretching, leading to an interruption of the axial flow. It will therefore break up. Another vortex is produced that follows the same dynamics and so on with a very well defined frequency.

Mechanisms behind the formation of vortices have been determined through visualizations. It has been observed that vorticity filaments were generated by the roll-up of a fluid sheet around the

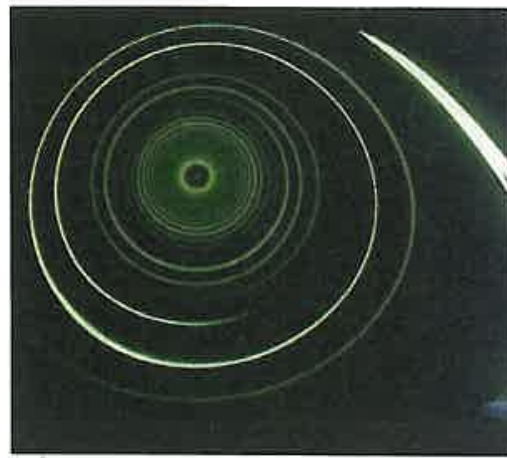

Fig. 4a: Visualization of the cross-section of a stretched vortex (plan $x, y$ on Figure 3). A sheet of fluorescein is injected just before stretching. Vortex formation by roll-up of fluid sheets on themselves is observed (image size: $3 \mathrm{~cm}$, vortex core diameter: $0.3 \mathrm{~cm}$ ).

vortex core.

An important point that has been studied is the structure of the vortex (Fig. 4). Indeed, the localization of the stretching does not allow a simple model for this vortex, such as what has been proposed when the stretching is uniform (Burgers' vortex). The axial component of the velocity must depend on the distance to the vortex axis $r$ (Fig. 4b). A model will be proposed very soon.

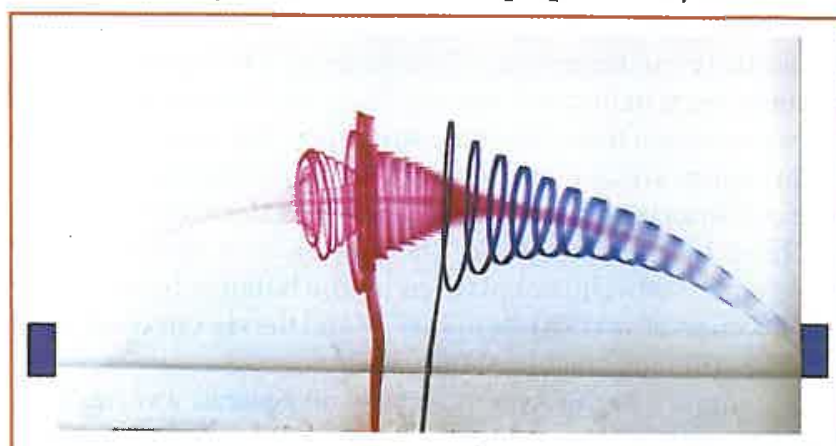

Aig. 4 b: Visualization of a vortex by injection of two small dye jets. Top view ( $p$ lan $x, z$ of Figure 3 ;. Flow comes from the bottom of the image towards the top and is stretched through suction represented as two small rectangles on the image (size of the image: $12 \mathrm{~cm}$ ).

PIV technique (Particles Image Velocimetry) was used for measurements of the velocity field in a transverse cross section of the vortex $(r, \theta)$. This technique consists of seeding the flow with small particles that are traced for the determination of the velocity fields. Two successive images of the $2 \mathrm{D}$ cross-section of the flow are recorded with a video camera. These images are usually obtained thanks to a laser sheet illuminating only particles within its light sheet. The velocity field is then evaluated from the two successive images by following the displacements of particles.

Figures 5 gives an example of what can be obtained with this technique.

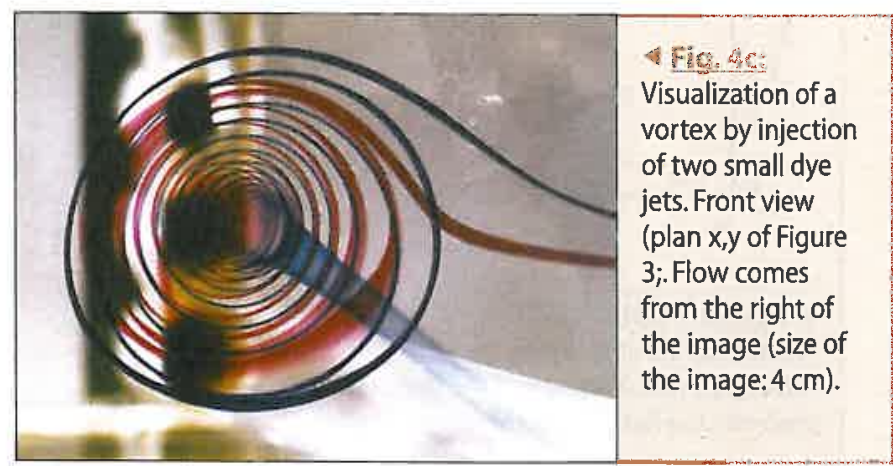



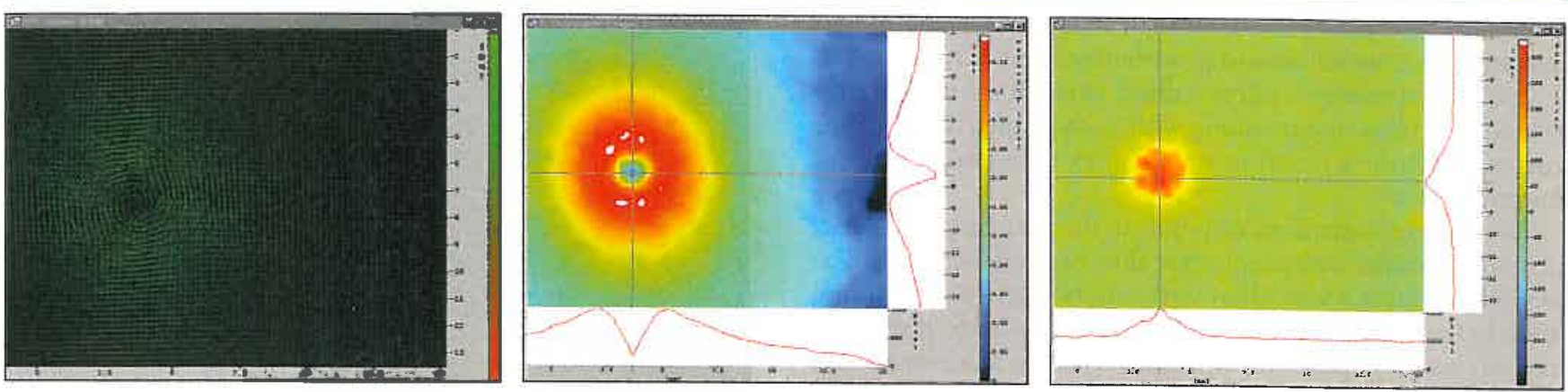

Thanks to different experimental techniques available in our laboratory, we were able to measure quantitatively the evolution of the vortex characteristics as a function of the stretching $\gamma$. Main results are summarized in the following figures:

Figure 6a plots the amplification of the initial vorticity $\omega_{f} / \omega_{i}$ as a function of the stretching. The initial vorticity $\omega_{i}$ was deduced from the velocity profile just before stretching and the final vorticity $\omega_{f}$ is evaluated from the velocity profile of the vortex. This experiment enables a large range of parameters to be explored as the vorticity can be enhanced by a factor of 100 ! Figure $6 \mathrm{~b}$ shows the maximum azimuthal velocity $V_{\theta \max }$ and the mean radius of the vortex $r_{0}$ as a function of the stretching. The results presented in this figure are obtained from PIV measurements technique. One can observe that $r_{0}$ strongly depends on the stretching and is not fixed by the diameter of the suction hole. It follows that $r_{0} \propto(v / \gamma)^{1 / 2}$ which is explained by the balance between the amplification of vorticity term $(\omega \nabla) V$ and the viscous dissipation term $v \Delta \omega$ in the equation of the vorticity.

In summary, in this experiment we can generate a vortex with characteristics of $V_{\theta \max } \approx 1 \mathrm{~cm} / \mathrm{s}$ and $2 r_{0} \approx 1 \mathrm{~cm}$ for a stretching just large enough to produce a vortex. For maximum stretching, just before the vortex becomes too unstable and explodes in a tur-

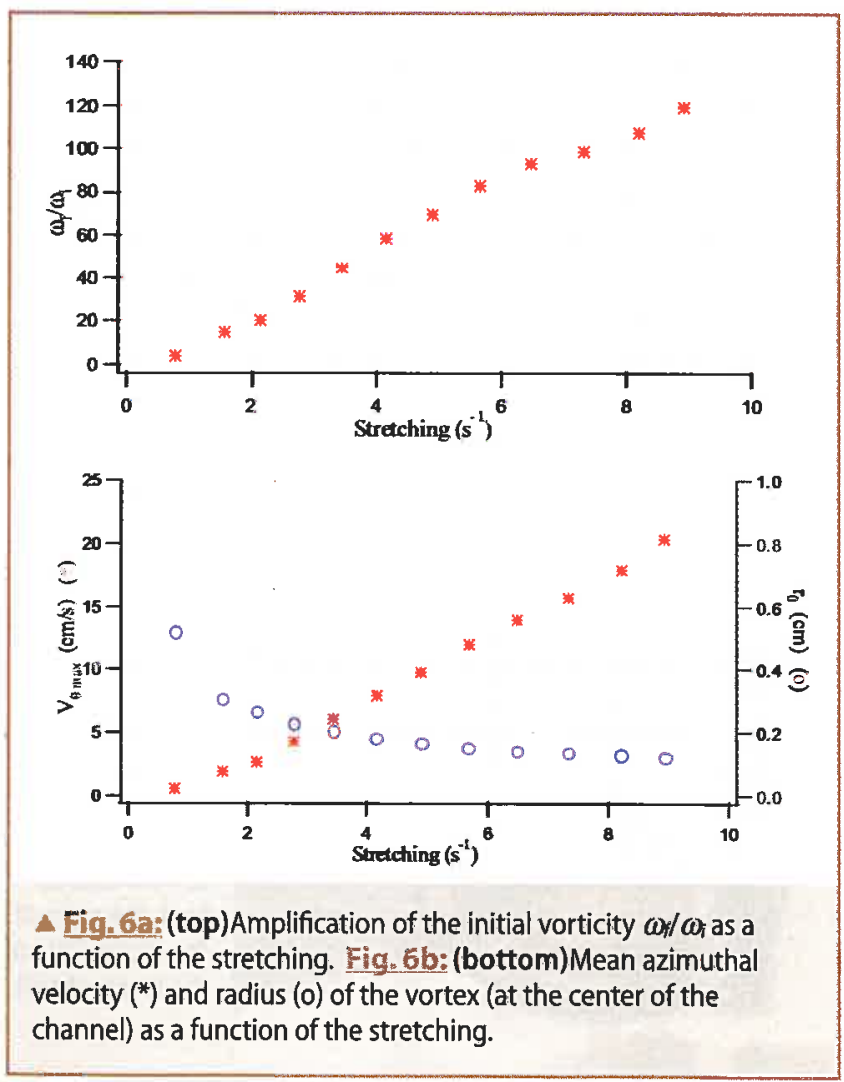

A. Fig. 5a: (left) Velocity field obtained by PIV on a cross-section of the vortex (plan $x, y)$. Fig. 5 b: (centre) Velocity modulus that is approximately the azimuthal velocity $V_{\theta}$ the radial velocity $V_{r}$ being much smaller than $V_{\theta}$. The shape of the vortex (its ellipticity) can be determined with these measurements. Red color coresponds to the largest velocity $\left(v_{\theta} \max \right)$ that is reached for $r \approx r_{0}$, while blue color correspond to the slowest velocity. Modulus of velocity profiles are shown on the sides of the image, with maxima at $r \approx r_{0}$ for $v \approx v_{\theta \text { max }}$ and a minimum at the center of the vortex where radial and azimuthal velocities are almost zero. Fig. $5 \mathrm{ci}$ (right)The curl of the velocity gives the axial vorticity. Particularly, one observes that vorticity is concentrated in the vortex core, and that the flow is quasi irrotational around. This calculation has been performed after a Gaussian-type filtering of the velocity field of Figure $5 \mathrm{a}$.

bulent burst, the vortex characteristics become as large as $V_{\theta \max } \approx 20 \mathrm{~cm} / \mathrm{s}$ with a diameter $2 r_{0} \approx 0.2 \mathrm{~cm}$.

Indeed, we observe that the more the vortex is stretched, the more its vorticity is amplified, the more its diameter decreases, and the faster it rotates; the circulation being the only quantity that is conserved. However, note that in this experiment, the circulation increases, but for another reason: when stretching is increased by increasing the flow rate of the suction, entrance conditions are modified as well, and the injected initial vorticity becomes more important. dynamics is observed. In that case, the flow tends to advect the turbulent burst. Mechanisms leading to this turbulent explosion are very complex and depend on different kind of instabilities.

\section{Experiment between two co-rorating discs}

Another experiment devoted to the study of stretched vortices consists of stretching the vorticity of a flow between two corotating discs: The discs of diameter $2 R(5,10$, or $15 \mathrm{~cm})$ are positioned in front of each other with a gap $d(0<d<20 \mathrm{~cm})$ between the disc surfaces. The discs can rotate at identical speeds

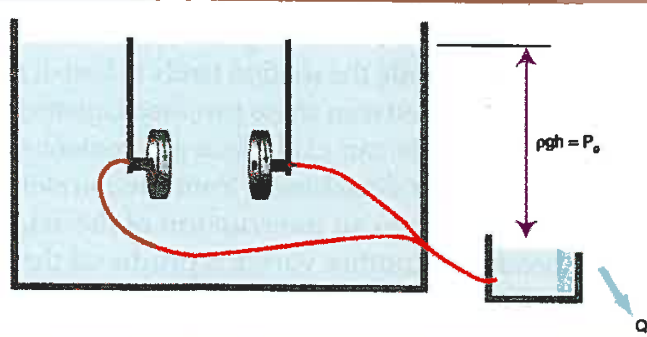

A Fig. 7: Experimental set-up.
When the flow rate downstream is not zero, a different vortex. Above a threshold, the vortex is unstable and explodes as a 


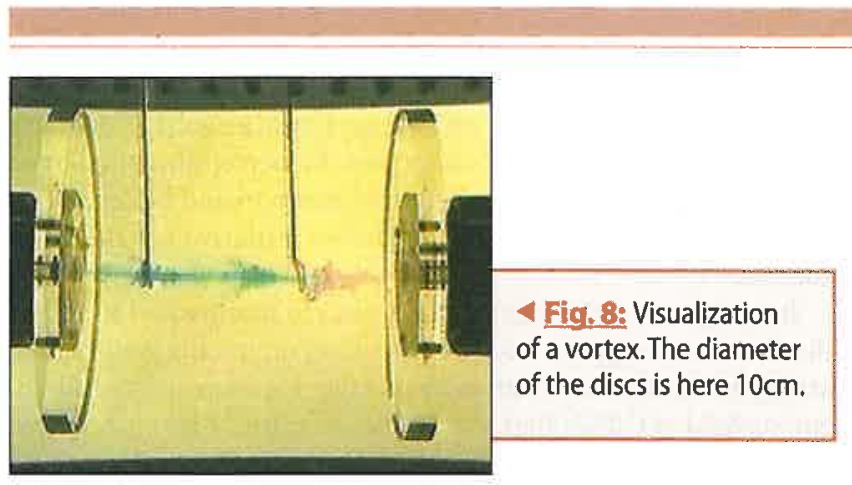

$\Omega(0<\Omega<1200 \mathrm{rpm})$. At the centre of each disc, a $0.5 \mathrm{~cm}$ diameter hole allows for suction thanks to a pressure drop (see Fig. 7). This set-up is placed in a water tank whose walls are at least 20 $\mathrm{cm}$ away. A vortex is then produced through the vorticity injected by the rotation of the discs and enhanced by stretching (Fig. 8).

Unlike the experiments of the straight channel, the two main control parameters (rotation and stretching) are independent here. Furthermore, the injected vorticity is much larger than in the experiment of the straight channel. These two experiments represent then a very large range of situations.

Pressure is an important quantity that is very difficult to measure in a vortex. We have shown that a probe, even very small $(200 \mu \mathrm{m}$ diameter) strongly destabilize the vortex. The mean pressure in the vortex has been deduced from the measurements of the feedback of the rotation on the stretching: As illustrated in Figure 8 , the stretching is produced by a suction system based on a pressure drop between the water level in the big tank (that is maintained at a constant level), and the water level of a small tank connected to the suction slots. The flow rate is measured when the discs are at rest and there is no vortex. A vortex is generated a few seconds after the discs start to rotate with the conditions that the stretching, the distance between the discs, the rotation speed etc are suitable. One observes a flowrate drop in the small tank. This is explained by the presence of the vortex which induces a pressure drop in its core. This pressure drop counterbalances the suction and the stretching, hence the flowrate of the small tank. From the flow rate drop, we evaluate the mean pressure drop in the vortex core: The flow rate with a vortex and a difference of water-level $h 1$ between the two tanks, is the same as that without a vortex but with a smaller difference of water-level

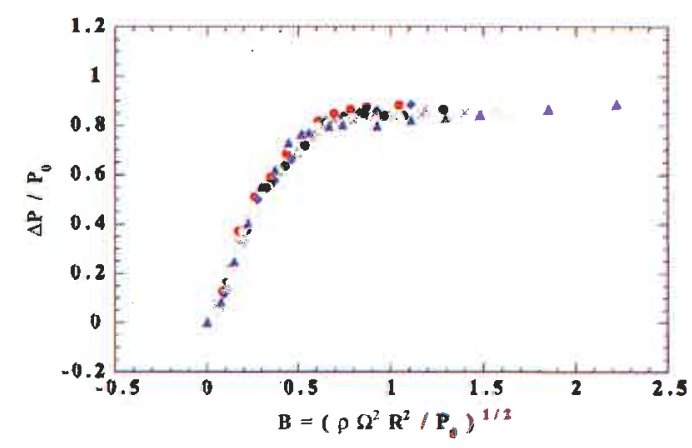

4 Fig. 9: Pressure drop into the vortex $P / P_{0}$ where the initial pressure drop $P_{0}$ is imposed by the difference between the water level in the two tanks when there is no vortex $(\Omega=0)$ as a function of a parameter $B$ that represents the ratio between the pressure drop generated by the rotation and $P_{0}$. Saturation proves that above a threshold, rotation lost its efficiency.
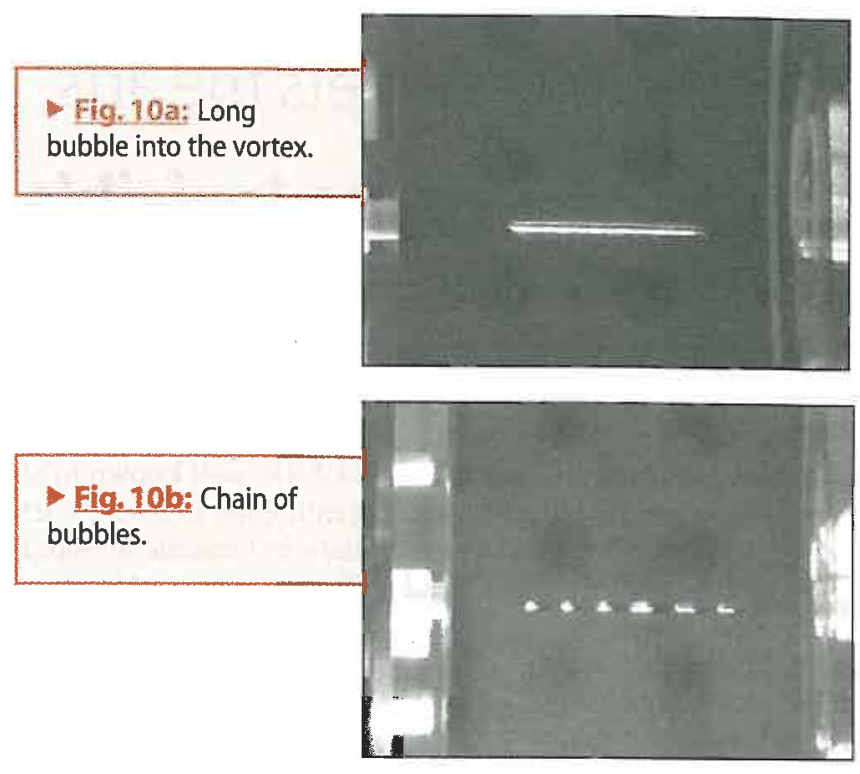

$h 2$. The pressure drop $P$ in the vortex core is given by the relation $P v=\rho g \Delta h$, where $\Delta h=h 1-h 2$. This pressure drop is measured as a function of the initial stretching (i.e. $h 1$ ), the size $R$ of the discs, the distance $d$ between the discs and the rotation velocity $\Omega$ (Fig. 9). This study has been performed with F. Moisy and B. Guard.

The pressure drop in the vortex can be large enough to trap micro bubbles naturally present in the water of the tank. This can lead to the formation of a long bubble that stays along the vortex core (Fig. 10a). The bubble is observed to break up into regularly spaced smaller bubbles along the vortex axis (Fig. 10b). 'This break up is due to the Rayleigh instability.

An interesting point is that, even if the flow rate in the suction holes is of the order of a few $1 / \mathrm{min}$, with an axial velocity of a few $\mathrm{m} / \mathrm{s}$, the pressure drop in the vortex core is large enough to maintain the bubbles.

Note that it is not cavitation that is observed, as it would require the pressure in the vortex core to be smaller than the vapor pressure of the water. The observations show only concentration of micro-bubbles naturally disolved in water.

The study of stretched vortices is a challenging research topic that presents lots of experimental difficulties due to large velocity gradients, three dimensionality and unsteadyness. However, we believe that mechanisms studied in these "standard" vortices are the same as those of filaments of vorticity in turbulent flows. We hope in that way to contribute to a better understanding and modeling of turbulence, with flow control and prediction as our main objectives. Flow control is indeed an important challenge for the future, in order to reduce the energy losses in turbulent flows (drag reduction, better efficiency in mixing,...). Prediction is also an important challenge for instance in flow control (better prediction leads to better control), or in weather forecasting.

\section{Footnotes}

${ }^{1}$ This article is based on an original version published in the Bulletin de la SFP (French Physical Society), 132, p.4, 1 dec. 2001

${ }^{2} \mathrm{~A}$ boundary layer is a thin layer that develops over a wall and connects the wall where the velocity is zero and the free-stream flow. It is inside this layer that viscosity plays a role whereas it has a much smaller effect in the rest of the flow. This region corresponds to the zone where the velocity gradient produces vorticty. 